\title{
FIBRES OF HUREWICZ AND APPROXIMATE FIBRATIONS
}

\section{S. HUSCH ${ }^{1}$}

A mapping $p: E \rightarrow B$ is a Hurewicz fibration if given mappings $H$ : $X \times[0,1] \rightarrow B$ and $h: X \rightarrow E$ such that $p h(x)=H(x, 0)$ for all $x \in X$, then there exists $G: X \times[0,1] \rightarrow E$ such that $p G=H$ and $G(x, 0)=h(x)$ for all $x \in X$. If $x \in B$, then $p^{-1}(x)$ is called a fibre of $p$. Suppose that $p: E \rightarrow B$ is a Hurewicz fibration between closed connected manifolds of dimensions $m$ and $n$, respectively. If $E, B$ and $p$ are smooth, then $p$ is locally trivial and the fibres are smooth manifolds of dimension $m-n$. If $p$ is not smooth, then $p$ need not be locally trivial nor need the fibre be a manifold [9]. In [12], F. Raymond showed that the fibre must be a generalized manifold. One of the main results of this paper is the following.

THEOREM 1. Let $p: E \rightarrow B$ be a Hurewicz fibration from a closed connected $m$ dimensional CAT manifold onto an $n$-dimensional TOP manifold with fibre $F$. If $m-n \geqq 5$ and if $\mathrm{Wh}\left(\pi_{1}(F) \oplus \mathrm{Z}^{r}\right)=0$ for all $r \leqq n-1$, then the fibre of $p$ has the homotopy type of a closed CAT manifold of dimension $m-n$.

Wh $\left(\pi_{1}(F) \oplus Z^{r}\right)$ denotes the Whitehead group [11] of the direct sum of the fundamental group of $F$ and the free Abelian group of rank $r$. Recall that if $F$ is simply connected or has fundamental group isomorphic to a free Abelian group, then $\mathrm{Wh}\left(\pi_{1}(F) \oplus \mathrm{Z}^{r}\right)=0$ for all $r$ [11]. CAT denotes one of the three categories: $\mathrm{DIFF}=$ differentiable, $\mathrm{PL}=$ piecewise linear or $\mathrm{TOP}=$ topelogical.

A mapping $p: E \rightarrow B$ is an approximate fibration if given an open cover $\mathscr{U}$ of $B$ and mappings $H: X \times[0,1] \rightarrow B$ and $h: X \rightarrow E$ such that $p h(x)=H(x, 0)$ for all $x \in X$, then there exists $G: X \times[0,1] \rightarrow E$ such that $p G$ and $H$ are $\mathscr{U}$-close (i.e., given $(x, t) \in X \times[0,1]$, then there exists $U \in \mathscr{U}$ such that $p G(x, t)$ and $H(x, t)$ are elements of $U)$ and $G(x, 0)=h(x)$ for all $x \in X$. Coram and Duvall [3] introduced the concept of approximate fibrations and showed that they have many similar properties as Hurewicz fibrations if one uses shape-theoretic

\footnotetext{
${ }^{1}$ Research was conducted while the author was on leave at the University of Zagreb and was supported under an exchange program between the National Academy of Sciences (U.S.A.) and the Yugoslav Council of Academies.
}

Received June 1, 1977. 
concepts in place of their homotopy-theoretic counterparts. For example, if $p: E \rightarrow B$ is an approximate fibration between compact ANR's and if $B$ is connected, then each fibre is an FANR and any two fibres have the same shape. Coram and Duvall construct an approximate fibration from the torus to the 1sphere such that all the fibres except one are 1-spheres and the exceptional fibre is a Warsaw circle.

THEOREM 2. Let $p: E \rightarrow B$ be an approximate fibration from a closed connected m-dimensional CAT manifold onto an n-dimensional TOP manifold with fibre $F$. If $m-n \geqq 5$, Wh $\left(\check{\pi}_{1}(F) \oplus Z^{r}\right)=0$ for $r \leqq n$, and if $F$ has the shape of $a$ finite complex, then $F$ has the shape of a closed CAT manifold of dimension $m-n$.

$\check{\pi}_{1}(F)$ denotes the shape fundamental group [1] of $F$.

The author does not know whether the hypotheses on the fibre are necessary. However, it should be noted that S. Ferry [6] has constructed an approximate fibration from a compact ANR onto the 1-sphere such that the fibre does not have the shape of a finite complex.

First, we recall some results from the thesis of L. C. Siebenmann [13]. Although these results are stated in the DIFF category, they are also valid in any of the three categories CAT = DIFF, PL or TOP by the work of Kirby and Siebenmann [10]. Let $M$ be an open connected $\dot{r}$-dimensional CAT manifold with a finite number of ends $\varepsilon_{1}, \varepsilon_{2}, \ldots, \varepsilon_{t}$. The end $\varepsilon_{i}$ is stable if there exists a sequence of connected neighborhoods $\left\{U_{i}\right\}$ of the end $\varepsilon_{i}$ such that $U_{i} \supseteqq U_{i+1}$ for all $i, \bigcap_{i} \operatorname{cl}\left(U_{i}\right)=\varnothing$, and if $\alpha_{i}: \pi_{1}\left(U_{i+1}, x_{i+1}\right) \rightarrow \pi_{1}\left(U_{i}, x_{i}\right)$ denotes the homomorphism induced by inclusion and a path between $x_{i+1}$ and $x_{i}$, then $\alpha_{i} \mid$ image $\alpha_{i+1}$ : image $\alpha_{i+1} \rightarrow$ image $\alpha_{i}$ is an isomorphism of finitely presented groups. The inverse limit of this sequence of groups is called the fundamental group of the end $\varepsilon_{i}$ and is denoted by $\pi_{1}\left(\varepsilon_{i}\right)$. A submanifold $V \cong M$ is called a 1neighborhood of the end $\varepsilon_{i}$ if $V$ is a closed connected neighborhood of $\varepsilon_{i}$ with compact connected boundary $\partial V$ such that the inclusions $\partial V \cong V$ and of the end $\varepsilon_{i}$ into $V$ induce isomorphisms of fundamental groups. Siebenmann shows that if $r \geqq 5$ and if the end $\varepsilon_{i}$ is stable, then $\varepsilon_{i}$ has arbitrarily small 1 -neighborhoods.

THEOREM 3 (Siebenmann). Let $M$ be an open connected CAT manifold of dimension $\geqq 6$ with a finite number of ends $\varepsilon_{1}, \varepsilon_{2}, \ldots, \varepsilon_{t} . M$ is homeomorphic to the interior of a compact CAT manifold if and only if

3.1 each end is stable;

3.2 each end has arbitrarily small 1-neighborhoods which are dominated by a finite complex;

3.3 for each $i=1,2, \ldots, t$, a certain invariant $\sigma_{i} \in \widetilde{K}_{0}\left(\pi_{1}\left(\varepsilon_{i}\right)\right)$ must be zero. 
The invariant $\sigma_{i}$ vanishes if and only if $\varepsilon_{i}$ has arbitrarily small 1neighborhoods which have the homotopy type of a finite complex.

Let $T^{r}$ denote the product of $r 1$-spheres.

TheOREM 4. Let $F$ be a compact connected ANR such that for some $r \geqq 0$, $F \times T^{r}$ has the homotopy type of a closed connected CAT $n$-manifold, $n \geqq r+5$. If $\mathrm{Wh}\left(\pi_{1}(F) \oplus Z^{i}\right)=0$ for $i \leqq r$, then $F$ has the homotopy type of a closed connected $(n-r)$-dimensional CAT manifold.

Proof. The proof will be by induction on $r$. The theorem is trivially true for $r$ $=0$. Let $f: V \rightarrow F \times T^{r}$ be a homotopy equivalence of a closed connected CAT $n$-dimensional manifold onto $F \times T^{r}$. Since $W h\left(\pi_{1}(F) \oplus Z^{r}\right)=0$, the torsion [2] of $f$ is zero. Let $Q$ denote the Hilbert cube. By [14], $f \times$ id: $V \times Q \rightarrow F \times T^{r} \times Q$ is homotopic to a homeomorphism $h$.

Let $p: F \times R \times T^{r-1} \rightarrow F \times T^{r}$ be a covering map ( $R=$ real numbers) and let $q: \hat{V} \rightarrow V$ be the pull-back of $p$ by $f$. By covering space theory, there exists a homeomorphism $\tilde{h}: \hat{V} \times Q \rightarrow F \times R \times T^{r-1} \times Q$ such that $(p \times$ id $) \tilde{h}=h(q \times$ id $)$. Note that $\tilde{V}$ is a CAT manifold which is a proper deformation retract of $\tilde{V} \times Q$. Hence, $\tilde{V}$ has two ends $\varepsilon_{1}$ and $\varepsilon_{2}, \tilde{V}$ is stable at each end and $\pi_{1}\left(\varepsilon_{i}\right)$ is isomorphic to $\pi_{1}\left(F \times T^{r-1}\right)$. Let $V_{1} \subseteq \tilde{V}$ be a 1-neighborhood of one of the ends of $\tilde{V}$. Without loss of generality, we may assume that there exists $a \in \mathbf{R}$ such that $F \times[a,+\infty) \times T^{r-1} \times Q \subseteq \operatorname{int} \tilde{h}\left(V_{1} \times Q\right)$. Let $C$ be the compact subset of $F \times R \times T^{r-1} \times Q$ whose frontier is $\left(F \times\{a\} \times T^{r-1} \times Q\right) \cup \tilde{h}\left(\partial V_{1} \times Q\right)$. Note that $C$ is a deformation retract of $\tilde{h}\left(V_{1} \times Q\right)$ and since $C$ is a compact ANR, $V_{1}$ has the homotopy type of a finite complex [15]. By Theorem 3. $\tilde{V}$ is homeomorphic to the interior of a compact CAT manifold $W$. If $W_{1}$ is a component of $\partial W$, then it is straightforward to check that the inclusion of $W_{1}$ into $W$ is a homotopy equivalence. Hence $F \times T^{r-1}$ is homotopy equivalent to the closed connected CAT manifold $W_{1}$. The induction hypothesis implies that $F$ has the homotopy type of a closed, connected CAT manifold of dimension $n-r$.

Proof of theorem 1. Suppose $F$ is not connected. Since $F$ is a compact ANR, $F$ has a finite number of components. Hence, from the long exact homotopy sequence of a fibration, $p_{*}\left(\pi_{1}(E)\right)$ has finite index in $B$. Let $\tau: \widetilde{B}$ $\rightarrow B$ be the covering space corresponding to $p_{*}\left(\pi_{1}(E)\right)$. Let $\tilde{p}: \tilde{E} \rightarrow \tilde{B}$ be the pull-back of $p$ by $\tau$. It is easily checked that $\widetilde{E}$ and $\widetilde{B}$ are compact manifolds, $\tilde{p}$ is a Hurewicz fibration the fibre of $\tilde{p}$ is homeomorphic to a component of $F$ and each component of $F$ is some fibre of $\tilde{p}$. Thus it suffices to consider the case when $F$ is connected.

Let $U_{0}$ be an open $n$-cell in $B$ and let $U \subseteq U_{0}$ be an open subset which is homeomorphic to $T^{n-1} \times R$. Let $W=p^{-1}(U)$; note that $W$ is an open 
connected CAT manifold with two ends. By [5], there exists a fibre homotopy equivalence $p^{-1}\left(U_{0}\right) \rightarrow F \times U_{0}$; consider the restriction $\alpha: W \rightarrow F \times U$ of this fibre homotopy equivalence to $W$. It is easily checked that $W$ is stable at each end and that the fundamental group of each end is isomorphic to $\pi_{1}\left(F \times T^{n-1}\right)$. Let $W_{1}$ be a 1-neighborhood of an end of $W$. Identify $U$ with $T^{n-1} \times \mathrm{R}$ such that there exists $a \in \mathrm{R}$ with $p^{-1}\left(T^{n-1} \times[a, \infty)\right) \subseteq W_{1}$. Analogous to the proof of the corresponding fact in the proof of Theorem 4, one can show that the compactum $C \cong W_{1}$ whose frontier (in $\left.W_{1}\right)$ is $p^{-1}\left(T^{n-1} \times\{a\}\right)$ is a deformation retract of $W_{1}$. Thus $C$ is a compact ANR and $W_{1}$ has the homotopy type of a finite complex [15]. By Theorem 3,W is homeomorphic to the interior of a compact CAT manifold $S$; let $V$ be a component of $\partial S$. Again, it is easily checked that the inclusion $V \cong S$ is a homotopy equivalence. Therefore $V$ is homotopy equivalent to $F \times T^{n-1}$ and the theorem follows from Theorem 4 .

Proof of Theorem 2. As in the proof of Theorem 1, it suffices to consider the case when the fibre is connected. Let $K$ be a finite complex which has the same shape as $F$. We now attempt to follow the proof of Theorem 1. Choose $U$, $U_{0}$ and $W$ as before; in general, there does not exists a fibre homotopy equivalence $\alpha: W \rightarrow F \times U$. However, by [8], there exists a proper homotopy equivalence $\beta: W \rightarrow K \times U$. Hence, again it is easily checked that $W$ is an open CAT manifold with two ends, $W$ is stable at each end and the fundamental group of each end is isomorphic to $\pi_{1}\left(K \times T^{n-1}\right) \cong \check{\pi}_{1}\left(F \times T^{n-1}\right)$. Let $W_{1}$ be a 1 -neighborhood of an end of $W$ and choose $a \in \mathbb{R}$ with $p^{-1}\left(T^{n-1} \times[a\right.$, $+\infty)) \subseteq W_{1}$. Let $C$ be the compactum in $W_{1}$ whose frontier is $p^{-1}\left(T^{n-1} \times\{a\}\right)$; now $C$ need not be locally connected and, hence, we cannot proceed as before to show that $W$ satisfies the hypotheses of Theorem 3 .

However, we claim that $C$ is an FANR [1]. For, suppose that $a$ was chosen such that

$$
\partial W_{1} \cap p^{-1}\left(T^{n-1} \times(a-1, a)\right)=\varnothing .
$$

Let $B_{i}=p^{-1}\left(T^{n-1} \times(a-1, a+1 / i)\right)$ for each positive integer $i$. It follows from the long exact homotopy sequence of an approximate fibration [3] and the Whitehead theorem that the inclusion $B_{i+1} \cong B_{i}$ is a homotopy equivalence for each $i$. Hence $C \cup B_{i+1} \subseteq C \cup B_{i}$ is a homotopy equivalence for each $i$. Since $C$ $=\bigcap_{i}\left(C \cup B_{i}\right)$, this implies that $C$ has the pointed shape of a simplicial complex. Hence by [4], $C$ is an FANR.

By $[1 ;$ p. 254], $C$ is pointed shape dominated by a finite complex. Since the inclusion of $C \cup B_{i}$ into $W_{1}$ is a homotopy equivalence (by exactly the same argument showing that $B_{i+1} \cong B_{i}$ is a homotopy equivalence), the inclusion of $C$ into $W_{1}$ is a pointed shape equivalence. Hence $W_{1}$ is pointed shape dominated by a finite complex. Since $W_{1}$ is an ANR, this implies that $W_{1}$ is dominated by a finite complex $[1 ;$ p. 102]. 
The author is unable to show that $W_{1}$ has the homotopy type of a finite complex. However, by the product formula for Wall's obstruction [7], $W_{1} \times S^{1}$ $\left(S^{1}=1\right.$-sphere) has the homotopy type of a finite complex. By Theorem 3, $W \times S^{1}$ is homeomorphic to the interior of a CAT compact manifold $S$; let $V$ be a component of $\partial S$. Again, $V \cong S$ is a homotopy equivalence and, hence, $V$ is homotopy equivalent to $K \times U \times S^{1}$. Thus $V$ is homotopy equivalent to $K \times T^{n}$; by Theorem $4, K$ is homotopy equivalent to a closed connected $(m-n)$-dimensional CAT manifold. Since $K$ has the same shape as $F$, Theorem 2 follows.

\section{REFERENCES}

1. K. Borsuk, Theory of Shape, Monografie Matematyczne Tom 59, Polish Scientific Publishers, Warsaw 1975.

2. T. A. Chapman, Simple homotopy theory for ANR's, (to appear).

3. D. S. Coram and P. F. Duvall, Jr., Approximate Fibrations, (to appear).

4. D. Edwards and R. Geoghegan, The stability problem in shape and a Whitehead theorem in prohomotopy, Trans. Amer. Math. Soc. 214 (1975), 261-278.

5. E. Fadell, On fiber spaces, Trans. Amer. Math. Soc. 90 (1959), 1-14.

6. S. Ferry, Approximate fibrations with non-finite fibers, (to appear).

7. S. Gersten, A product formula for Wall's obstruction, Amer. J. Math. 88 (1966), 337-346.

8. R. Goad, Local homotopy properties of maps and approximation by fibre bundle projections, Thesis, University of Georgia, 1976.

9. L. S. Husch, Hurewicz fibrations need not be locally trivial, Proc. Amer. Math. Soc. 61 (1976), 155-156.

10. R. C. Kirby and L. C. Siebermann, Foundational Essays on Topological Manifolds, Smoothings and Triangulations, Annals of Mathematics Studies 88, Princeton University Press, Princeton, 1977.

11. J. Milnor, Whitehead torsion, Bull. Amer. Math. Soc. 72 (1966), 358-426.

12. F. Raymond, Local triviality for Hurewicz fiberings of manifolds, Topology 3 (1965), 43-57.

13. L. C. Siebenmann, The obstruction to finding a boundary for an open manifold of dimension greater than five, Thesis, Princeton University 1965.

14. J. West, Mapping cylinders of Hilbert cube factors, General Topology and its Appl. 1 (1971), 111-125.

15. J. West, Compact ANR's have finite types, Bull. Amer. Math. Soc. 81 (1965), 163-165.

UNIVERSITY OF TENNESSEE, KNOXVILLE, TENNESSEE 37916, U.S.A.

AND

UNIVERSITY OF ZAGREB, ZAGREB, YUGOSLAVIA 